\title{
PERFIL EPIDEMIOLÓGICO E OBSTÉTRICO DE GESTANTES COM SÍNDROME HELLP
}

\author{
Inez Sampaio Nery¹, Liliana Soares Viana², Lívia Maria Mello Viana ${ }^{3}$, Telma Maria Evangelista de Araújo ${ }^{4}$, \\ Verbênia Cipriano Feitosa ${ }^{5}$, Virgínia Félix Pereira ${ }^{2}$
}

\begin{abstract}
RESUMO: Estudo epidemiológico descritivo objetivou caracterizar as gestantes com Síndrome HELLP quanto aos dados sociodemográficos e obstétricos e levantar o número de casos dessa patologia. A população foi constituída por 14 gestantes com diagnóstico de Síndrome HELLP, confirmado por exames laboratoriais e avaliação clínica, e atendidas em Unidade de Cuidados Intensivo no período de janeiro de 2010 a dezembro de 2012. A coleta de dados ocorreu em 2013, com consulta aos prontuários, utilizando-se formulário com variáveis sociodemográficas e obstétricas. Os resultados evidenciaram que $64,3 \%$ das gestantes tinham acima de 26 anos; 92,9\% eram pardas; 64,3\% não realizaram o pré-natal; 78,6\% não tinham história de complicações em gestações anteriores. Os resultados apontam dados semelhantes a outros estudos sobre o perfil epidemiológico da Síndrome HELLP e servem de alerta sobre a carência de informações nos prontuários. DESCRITORES: Epidemiologia; Síndrome HELLP; Gestantes.
\end{abstract}

\section{PERFIL EPIDEMIOLÓGICO Y OBSTÉTRICO DE GESTANTES CON SÍNDROME HELLP}

RESUMEN: Estudio epidemiológico descriptivo cuya finalidad fue caracterizar las gestantes con Síndrome HELLP cuanto a los datos sociodemográficos y obstétricos y apuntar el número de casos de esa patología. La población fue constituida por 14 gestantes con diagnóstico de Síndrome HELLP, confirmado por exámenes laboratoriales y evaluación clínica, y atendidas en Unidad de Cuidados Intensivo en el periodo de enero de 2010 a diciembre de 2012. Los datos fueron obtenidos en 2013, con consulta a los prontuarios, utilizándose formulario con variables sociodemográficas y obstétricas. Los resultados evidenciaron que $64,3 \%$ de las gestantes tenían más de 26 años; $92,9 \%$ eran pardas; $64,3 \%$ no realizaron el prenatal; $78,6 \%$ no tenían historia de complicaciones en gestaciones anteriores. Los resultados apuntan datos semejantes a otros estudios sobre el perfil epidemiológico de la Síndrome HELLP y sirven de alerta sobre la carencia de informaciones en los prontuarios. DESCRIPTORES: Epidemiologia; Síndrome HELLP; Gestantes.

\section{EPIDEMIOLOGICAL AND OBSTETRIC PROFILE OF PREGNANT WOMEN WITH HELLP SYNDROME}

\begin{abstract}
This descriptive epidemiological study, with a quantitative approach, aimed to characterize the pregnant women with HELLP Syndrome regarding socio-demographic and obstetric data, and to ascertain the number of cases of this pathology. The population was constituted by 14 pregnant women diagnosed with HELLP Syndrome, confirmed by laboratory tests and clinical evaluation, and attended in an intensive care unit in the period January 2010 to December 2012. Data collection occurred in 2013, consulting the hospital records, using a form with socio-demographic and obstetric variables. The results evidenced that $64.3 \%$ of the pregnant women were aged over 26 years old; $92.9 \%$ were of mixed European, African and Indigenous ancestry; $64.3 \%$ did not undertake prenatal check-ups; and $78.6 \%$ had no history of complications in previous pregnancies. The results indicate data similar to that of other studies on the epidemiological profile of HELLP Syndrome, and serves as a warning regarding the shortage of information in the patient records.
\end{abstract}

DESCRIPTORS: Epidemiology; HELLP Syndrome; Pregnant women.

\footnotetext{
${ }^{1}$ Enfermeira. Doutora em Enfermagem. Professora da Universidade Federal do Piauí. Teresina-PI-Brasil ${ }^{2}$ Enfermeira. Centro de Ensino Unificado. Teresina-PI-Brasil

${ }^{3}$ Enfermeira. Mestre em Enfermagem. Fundação Municipal de Saúde de Teresina. Teresina-PI-Brasil

${ }^{4}$ Enfermeira. Doutora em Enfermagem. Professora da Universidade Federal do Piauí. Teresina-PI-Brasil

${ }^{5}$ Enfermeira. Mestre em Enfermagem. Professora do Centro de Ensino Unifcado de Teresina. Teresina-PI-Brasil
} 


\section{INTRODUÇÃO}

O período gestacional para cada mulher tem um significado especial, variando de acordo com as particularidades de cada pessoa, e pode ser prazeroso ou preocupante; a gravidez passa a ser preocupante quando está associada a problemas de ordem emocional e/ou física. Dentre os problemas específicos do período gestacional merece destaque a questão das doenças hipertensivas específicas da gestação, que tem sido discutido mundialmente, principalmente nos países subdesenvolvidos e em desenvolvimento, por ser uma das maiores causas de morte materna, tornando-se um problema de saúde pública. As doenças hipertensivas específicas da gestação são classificadas como pré-eclâmpsia, eclâmpsia e na sua forma mais grave, a Síndrome HELLP ${ }^{(1)}$.

Essa é uma grave complicação, descrita primeiramente em 1982 por Louis Weinstein o qual relatou 29 casos de pré-eclâmpsia em que houve trombocitopenia, hemólise intravascular evidenciada pelos achados no esfregaço de sangue periférico e alterações nos testes de função hepática. O pesquisador sugeriu que as mulheres que apresentassem esse quadro laboratorial fossem diferenciadas daquelas que eram classificadas com pré-eclâmpsia grave. Essa patologia, de grave repercussão materna e fetal, foi denominada Síndrome HELLP, um acrônimo dos três critérios estabelecidos para sua presença $(\mathrm{H}=$ hemoly-

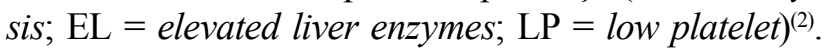

Neste contexto, é importante descrever o quadro clínico desta síndrome que tem como sinais e sintomas mais frequentes dor epigástrica - no quadrante superior direito, mal estar e náuseas. As formas leves podem passar despercebidas caso a correta avaliação laboratorial não seja feita. Apesar desse agravo à saúde possuir a sintomatologia das patologias hipertensivas, pode decorrer de causas diferentes e é bastante inespecífica, sendo este um dos principais problemas no seu diagnóstico. A evolução clínica da mulher com verdadeira Síndrome HELLP caracterizada por deterioração progressiva e súbita da condição materna e fetal, e associada a aumento da mortalidade e morbidade materna e perinatal ${ }^{(3)}$.

É recomendado que as gestantes com suspeita de pré-eclâmpsia realizem os testes laboratoriais apropriados para a triagem da Síndrome HELLP por meio de exames básicos para a triagem: hemograma completo com plaquetas, urinálise, creatinina sérica, desidrogenase lática (DHL), ácido úrico, bilirrubinas e transaminases; os testes mais específicos são reservados para aquelas mulheres com contagem de plaquetas abaixo de $100.000 / \mathrm{ml}^{(4)}$.
Embora seja menos frequente que a pré-eclâmpsia, o óbito materno varia de $1,1 \%$ em países desenvolvidos a $24 \%$ em países subdesenvolvidos. A intensidade da Síndrome HELLP, em média, pode acontecer em 24 a 48 horas após o parto; $31 \%$ podem ocorrer no pós-parto e, destas, $20 \%$ sem apresentar pré-eclâmpsia prévia, agravando ainda mais o quadro ${ }^{(5)}$. A Síndrome tem alta morbimortalidade; caracteriza-se como uma condição rara, que traz risco de morte, e que ocorre entre $0,2 \%$ a $0,6 \%$ das gestações. Algumas gestantes desenvolvem apenas uma ou duas das características da Síndrome ${ }^{(6)}$.

$\mathrm{O}$ tratamento representa grande desafio para obstetras em todo o mundo, recomenda-se que todas as pacientes com suspeita sejam hospitalizadas e as condutas imediatas consistem em antecipação do diagnóstico; avaliação das condições maternas e das condições fetais para identificar a necessidade de parto imediato, ou mais tardiamente; controle da pressão arterial; prevenção das convulsões com sulfato de magnésio; manejo de fluidos e eletrólitos; utilização criteriosa de sangue; manejo do trabalho de parto e parto; tratamento da gestante intensivamente no pós-parto; alerta para o desenvolvimento de falência sistêmica de múltiplos órgãos; e aconselhamento sobre gestações futuras ${ }^{(4)}$.

Apesar da baixa incidência, a alta morbidade/mortalidade materna e perinatal da Síndrome HELLP requer cuidados intensivos, e não deve ser desconsiderada a hipótese em qualquer paciente com pré-eclâmpsia, pois determinar atendimento eficiente durante a gestação é a única forma de estabelecer bom prognóstico materno e neonatal. Desta forma, em meio às considerações levantadas, objetivou-se caracterizar a população do estudo quanto aos dados sociodemográficos e obstétricos, levantar o número de casos de Síndrome HELLP na maternidade em estudo de 2010 a 2012, acreditando-se que este estudo possa contribuir para maior conhecimento acerca dessa Síndrome, pouco conhecida tanto entre profissionais que atuam na área, como entre gestantes

\section{MÉTODO}

A pesquisa configurou-se como um estudo epidemiológico descritivo, com abordagem quantitativa, desenvolvida em uma maternidade pública no Estado do Piauí, considerada de referência estadual, localizada na região sul da cidade de Teresina. A população constituiu-se por 14 gestantes com diagnóstico de Síndrome HELLP atendidas na Unidade de Cuidados Intensivo (UTI), no período de janeiro de 2010 a dezembro de 2012. O critério de inclusão foi o diagnóstico de Síndro- 
me HELLP confirmado por exames laboratoriais e avaliação clínica. A coleta de dados ocorreu em março de 2013. Realizou-se uma busca dos prontuários, a partir dos livros de registros e admissão das pacientes na referida UTI no período do estudo, com a hipótese de diagnosticar Síndrome HELLP; posteriormente realizou-se um levantamento da quantidade de pacientes admitidas no período em questão, usando o livro de admissão, e a partir daí, foi feito um percentual dos casos, triagem e análise retrospectiva daqueles com diagnóstico confirmado. Para sistematizar os dados foi utilizado um formulário-padrão, com questões predominantemente fechadas referentes às características epidemiológicas e a evolução clínica na população em estudo.

Os dados foram digitados utilizando o software Microsoft Office Excel versão 7.0. A apresentação dos achados foi feita por meio de tabelas e gráficos ilustrativos e a análise foi feita com base nas produções científicas mais atuais sobre a Síndrome HELLP.

O projeto de pesquisa foi autorizado pela Comissão de Ética sob protocolo n. 03105/2013 e pelo Comitê de Ética e Pesquisa da Faculdade Camilo Filho com CAAE n. 11921013.8.0000.5212. Foi oferecido ao representante da instituição cenário do estudo o termo de fiel depositário, conforme os princípios norteadores dispostos na Resolução $n^{\circ}$ 466/12, do Conselho Nacional de Saúde.

\section{RESULTADOS}

$\mathrm{Na}$ Tabela 1, os dados apontaram que a maioria $(64,5 \%)$ das gestantes apresentava idade de 26 anos e mais; $92,9 \%$ eram pardas; e quanto à escolaridade, $64,3 \%$ dos prontuários não havia esta informação.

Tabela 1 - Caracterização sociodemográfica das gestantes com Síndrome HELLP. Teresina-PI-Brasil, 2010-2012

\begin{tabular}{lcc}
\hline Variáveis & $\mathbf{n}$ & $\mathbf{\%}$ \\
\hline Faixa etária & & \\
Até 25 anos & 5 & 35,7 \\
$(\geq) 26$ anos & 9 & 64,3 \\
Cor & & \\
Parda & 13 & 92,9 \\
Negra & 1 & 7,1 \\
Escolaridade & & \\
Sem informação & 9 & 64,3 \\
Ensino fundamental & 3 & 21,4 \\
Ensino médio & 1 & 7,1 \\
Ensino superior & 1 & 7,1 \\
\hline Total & 14 & 100 \\
\hline
\end{tabular}

No Gráfico 1 observa-se que no ano de 2010, para cada 1041 gestantes internadas na UTI materna, 10 foram diagnosticadas com Síndrome HELLP, enquanto no ano de 2011 a cada 832 gestantes internadas na UTI materna, 12 apresentaram Síndrome HELLP e no ano de 2012 das 595 admissões na UTI materna, 18 foram diagnosticadas com a Síndrome.

Observa-se no Gráfico 2 que em 64,3\% dos prontuários não havia informações sobre a realização, ou não, do pré-natal.

Na Tabela 2 observa-se que grande parte das gestantes do estudo não apresentou complicações e óbitos fetais em gestações anteriores.

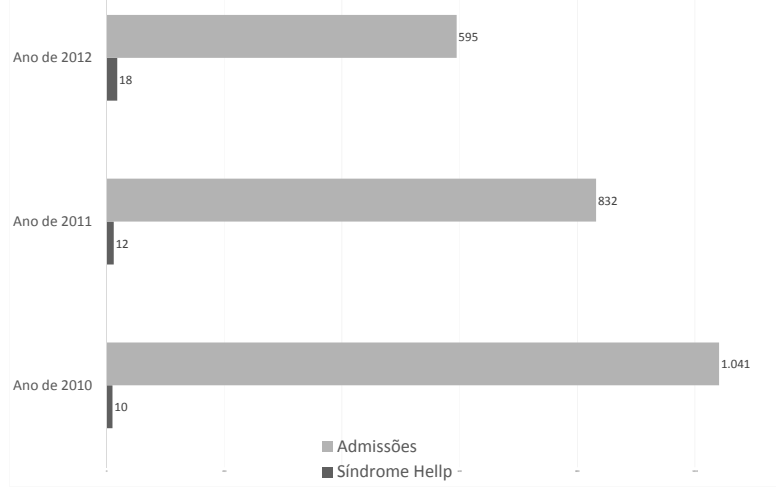

Gráfico 1 - Número de casos de Síndrome HELLP em relação ao total de internações no período. Teresina, 2010-2012

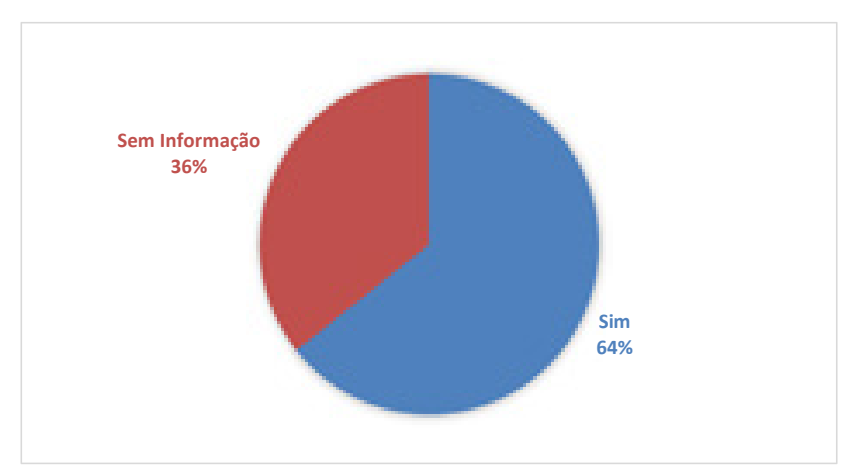

Gráfico 2 - Distribuição das mulheres com Síndrome HELLP segundo a realização do pré-natal. Teresina, 2010-2012

Tabela 2 - Caracterização das mulheres com Síndrome HELLP segundo os dados clínicos perinatais. Teresina-PIBrasil 2010-2012

\begin{tabular}{lcc}
\hline Variáveis & $\mathbf{n}$ & $\mathbf{\%}$ \\
\hline Complicações & em gestações anteriores \\
Sim & 3 & 21,4 \\
Não & 11 & 78,6 \\
Número de óbitos fetais em & gestações anteriores \\
Sim & 1 & 7,1 \\
Não & 13 & 92,9 \\
\hline
\end{tabular}




\section{DISCUSSÃO}

A maioria das gestantes com Síndrome HELLP foi composta por mulheres com 26 anos a mais, resultado semelhante a estudo realizado na cidade de Porto - Portugal (primigestas com média de idade de 28,5 anos) e em UTI Obstétrica de Recife (26,7 anos). A média de idade das pacientes com esta patologia variou de 24 a 30 anos, sendo, de modo geral, discretamente maior que a de mulheres com distúrbios hipertensivos sem Síndrome HELLP ${ }^{(3,7)}$.

Neste estudo a maioria das mulheres era parda, no entanto, uma pesquisa realizada no Hospital Universitário de Maringá mostrou que grande parte das gestantes era de cor branca ${ }^{(8)}$. Embora qualquer gestante possa desenvolver a Síndrome, algumas apresentam maior risco, incluindo aquelas de cor branca, com mais de 25 anos de idade, multíparas, com hipertensão arterial sistêmica e quadro de pré-eclâmpsia ou eclampsia. A análise da raça e da cor sempre foi uma missão difícil, levando-se em conta a dificuldade de entendimento para a classificação, havendo defesa sobre a não classificação ${ }^{(9)}$.

Diante do exposto, e sabendo que a diversidade étnica é a identidade marcante do Brasil, pode-se ter um retrato distorcido da verdadeira realidade social, interferindo e dificultando a análise concreta da população, e de seu perfil de morbimortalidade. De acordo com dados do Instituto Brasileiro de Geografia e Estatística, no Brasil da primeira década do Século XXI destacou-se uma mudança na distribuição da população, segmentada por cor ou raça, o que confirma uma tendência já detectada. Os dados da Pesquisa Nacional por Amostra de Domicílios mostram crescimento da proporção da população que se declara preta ou parda nos últimos dez anos: respectivamente, 5,4\% e 40\% em 1999; e 6,9\% e 44,2\% em 2009. Provavelmente, um dos fatores para esse crescimento é uma recuperação da identidade racial, já comentada por estudiosos do tema ${ }^{(10)}$.

Com relação aos dados da Tabela 1, resultado semelhante foi encontrado no estudo sobre mortalidade materna no Brasil, no qual em 5.603 registros não havia informação sobre a escolaridade; ou seja, 33,9\% dos óbitos registrados ${ }^{(11)}$. A realidade educacional do Nordeste é considerada baixa quando comparada à das Regiões Sul e Sudeste. Enquanto a Região Nordestina possui, para pessoas de 15 anos ou mais, 19,4\% de analfabetos e $31,6 \%$ de analfabetos funcionais, indicadores que correspondem, respectivamente, a 5,4\% e
$16,2 \%$ para o Sul e a $5,8 \%$ e $15,8 \%$ para o Sudeste. No Nordeste, a situação de estados como Alagoas, Piauí e Paraíba encontra-se ainda mais precária, com cerca de um quarto de suas populações com 15 ou mais anos analfabetas ${ }^{(12)}$.

A análise do percentual de casos de Síndrome HELLP demonstrou que das 2.468 mulheres que deram entrada na UTI materna, 40 casos foram confirmados como a Síndrome, o que representa $1,6 \%$ do total das patologias atendidas no período de 2010 a 2012 (Gráfico 1). Resultados parecidos foram evidenciados em estudo realizado com 30 puérperas acometidas por Síndrome HELLP, demonstrando que dos pacientes 7,5\% apresentaram Doença Hipertensiva Específica da Gestação (DHEG) e 4,7\% evoluíram para complicações pela síndrome ${ }^{(13)}$.

Um estudo realizado com entre 741 gestantes portadoras de DHEG admitidas entre janeiro a dezembro de 2009 evidenciou que 18 apresentaram a Síndrome HELLP, o equivalente a 2,42\% ${ }^{(14)}$. Essa Síndrome acomete $4 \%$ a $12 \%$ de gestantes com pré-eclâmpsia ou eclampsia, e se relaciona a altos índices de morbimortalidade materno-fetal ${ }^{(4)}$.

As síndromes hipertensivas na gestação são doenças de alta prevalência, e acarretam marcantes repercussões na morbimortalidade materna e perinatal. No Brasil, estas síndromes, principalmente nas suas formas graves, como a eclâmpsia e as complicações por Síndrome HELLP, são as principais causas de morte materna. Além disso, determinam significativo aumento da morbidade e mortalidade perinatais. A Síndrome HELLP constitui patologia no ciclo grávido puerperal, de alta morbimortalidade; uma condição rara, que traz risco de morte, e que ocorre entre $0,2 \%$ a $0,6 \%$ das gestações; entretanto algumas gestantes desenvolvem apenas uma ou duas das características desta Síndrome ${ }^{(9)}$.

No que diz respeito à realização do pré-natal, observou-se nos prontuários, que um grande percentual não apresentava informações (Gráfico 2), e os que apresentavam, estavam incompletas, não destacando o número de consultas realizadas. A captação precoce, fortalece a adesão da mulher ao acompanhamento sistemático e favorece o rastreamento adequado de eventuais fatores de risco. Portanto, o acompanhamento pré-natal deve ocorrer logo no início da gestação, ter cobertura universal, ser realizado de forma periódica, estar integrado com as demais ações preventivas e curativas e observar um número mínimo de consultas ${ }^{(15)}$. Os dados sobre mortalidade neonatal são considera- 
dos um dos principais indicadores para a avaliação dos serviços de saúde, sobretudo para a qualidade da assistência à gestante no pré-natal, parto, puerpério e cuidado neonatal ${ }^{(16)}$.

Adequado acompanhamento pré-natal prevê a captação da mulher com até 14 semanas (120 dias) de gestação, a realização de, no mínimo, seis consultas, sendo uma no primeiro trimestre, duas no segundo trimestre e três no terceiro trimestre. Desse modo, o acompanhamento pré-natal, sem dúvida, cumprirá com seu objetivo maior: a prevenção de morbidades e mortalidade perinatal ${ }^{(4)}$.

Dessa maneira, no tocante à investigação, os registros adequados proporcionam a descrição da evolução do paciente e de seu tratamento, fornecendo material para pesquisas de determinadas doenças e suas manifestações, além de subsidiarem a "busca ativa" nas investigações epidemiológicas. O diagnóstico precoce e o tratamento adequado são fundamentais para que seja possível alterar para melhor os resultados maternos e perinatais. A falta de informação nos prontuários pode implicar em riscos para mãe e para o feto, pois podem ser negligenciados exames e procedimentos terapêuticos, ou repetidos sem necessidade ${ }^{(4)}$.

$\mathrm{Na}$ análise dos dados obstétricos foi possível observar que a maioria das gestantes não apresentou complicações em gestações anteriores ou história de óbito fetal. As patologias hipertensivas podem decorrer de causas diferentes e é bastante inespecífica, além de variável, sendo este um dos principais problemas no diagnóstico da Síndrome HELLP; além disso, a maioria das pacientes não apresentou qualquer fator predisponente ${ }^{(3)}$. Assim, reconhece-se a importância do conhecimento dos antecedentes destas pacientes, visto que já é provada a associação entre a taxa de complicações e a presença patologias médicas prévias.

Embora a causa da Síndrome HELLP ainda não seja completamente explicada, pode levar à insuficiência cardíaca, hematoma hepático, insuficiência renal aguda, acidente vascular cerebral, sendo a hemorragia cerebral a principal causa de morte materna, responsável por $60 \%$, seguida de edema agudo do pulmão, e descolamento prematuro de placenta (DPP), resultando em morte fetal ${ }^{(17)}$.

A identificação de riscos e marcadores biológicos, precocemente, é fundamental para a prevenção ou redução dos riscos de mortalidade materna e fetal ${ }^{(4)}$. Esta prevenção é possível na consulta pré-natal, entretanto os profissionais que realizam o pré-natal precisam estar sensíveis a esta situação, e também ter preparo técnico para realizar triagem satisfatória das gestantes de alto risco. Portanto, a equipe de saúde, da qual o enfermeiro é integrante, deve estar atenta a todos os acontecimentos e dúvidas da gestante, buscando amenizar seu sofrimento através da orientação e ajuda. É necessário que o profissional enfermeiro possua conhecimento e sensibilidade, os quais são imprescindíveis para identificar, compreender e acompanhar alterações nos processos fisiológicos, patológicos e emocionais que permeiam a gestação de alto risco ${ }^{(9)}$.

Considerando que muitos prontuários estavam incompletos, esta pode ser uma limitação da pesquisa. No entanto, os questionamentos que nortearam o estudo foram elucidados ao longo da investigação, não havendo prejuízo ao alcance dos objetivos deste estudo.

\section{CONSIDERAÇÕES FINAIS}

Este estudo permitiu avaliar a situação da Síndrome HELLP e demonstrou perfil de mulheres pardas, acima de 26 anos e com baixa escolaridade. Constatou-se que, apesar de ser uma patologia pouco frequente, se desenvolve na maioria das vezes no terceiro trimestre de gestação.

Salienta-se a precariedade dos registros nos prontuários, tais como dados sociodemográficos, obstétricos e das consultas de pré-natal, evidenciando-se negligência uma vez que estas informações são necessárias durante todo o processo de internamento e pós-internamento da gestante.

Os resultados deste estudo apontam para a necessidade de realização de outros trabalhos de investigação para a melhor compreensão da problemática da Síndrome HELLP e permite alertar para o problema na carência no registro de informações essenciais. Embora não constitua objeto do estudo, foi possível observar a ausência da Sistematização da Assistência de Enfermagem, a qual contribuiria para o registro de informações e promoção da qualidade da assistência às pacientes.

\section{REFERÊNCIAS}

1. Pereira MN, Montenegro CAB, Rezende Filho J. Síndrome HELLP: diagnóstico e conduta. Femina. [Internet] 2008;36(2) [acesso em 25 mar 2013]. Disponível: http://www.febrasgo.org.br/site/wp-content/ uploads/2013/05/Femina-2_fevereiro_111-116.pdf

2. Montenegro CAB. Rezende J, obstetrícia fundamental. $12^{\mathrm{a}}$ ed. Rio de Janeiro: Guanabara Koogan; 2012.

3. Freitas DSF. Características clínicas, sintomatologia, 
tipo de parto, tratamento, morbilidade e mortalidade materna associada ao síndromehellp no chp-hsa: Estudo retrospectivo [dissertação]. Porto (PT): Instituto Ciências Biomédicas Abel Salazar; 2009.

4. Ministério da Saúde (BR). Departamento de Atenção Básica. Atenção ao pré-natal de baixo risco. Brasília: Conselho Nacional de Saúde; 2012.

5. Leão MD. Redução da morbi-mortalidade na síndrome de hellp completa com o uso de altas doses de dexametasona [tese]. Natal (RN): Universidade Federal do Rio Grande do Norte; 2008.

6. Ziegel EE, Cranley MS. Enfermagem obstétrica. $8^{\mathrm{a}}$ ed. Rio de Janeiro: Interamericana; 2008.

7. Leila K, Melania MRA, Giselly VM, João LPS. Perfil clínico, laboratorial e complicações de pacientes com síndrome HELLP admitidas em uma unidade de terapia intensiva obstétrica. Rev. Bras. Ginecol. Obstet. [Internet] 2008;30(2) [acesso em $06 \mathrm{abr}$ 2013]. Disponível: http://dx.doi.org/10.1590/S010072032008000200006

8. Angonesi J, Polato A. Doença Hipertensiva Específica da Gestação (DHEG), incidência à evolução para a Síndrome de HELLP [dissertação]. Maringá (PR): Centro Universitário de Maringá (CESUMAR); 2007.

9. Oliveira RS, Matos IC, Silva TBP, Azevedo NM, Andrade M, Espírito Santo FH. Síndrome Hellp: estudo de revisão para o cuidado de enfermagem. Enferm. Glob. [Internet] 2012;11(28) [acesso em 20 mai 2013]. Disponível: http://scielo.isciii.es/scielo.php?pid=S1695 61412012000400018\&script $=$ sci_arttext

10. Instituto Brasileiro de Geografia e Estatística (IBGE). Indicadores sociodemográficos e de saúde do Brasil 2010. Rio de Janeiro; 2010.

11. Ferraz L, Bordignon M. Mortalidade materna no Brasil: uma realidade que precisa melhorar. RBSP [Internet] 2012;36(2) [acesso em 15 fev 2013]. Disponível: http:// files.bvs.br/upload/S/0100-0233/2012/v36n2/a3253.pdf

12. Bezerra FD, Carvalho JS. Indicadores socioeconômicos do Nordeste: análise comparativa inter-re $\neg$ gional. Fortaleza: Banco do Nordeste do Brasil; 2010.

13. Oliveira MIV, Vasconcelos SG. Puérperas com síndrome de HELLP: análise baseada nos aspectos obstétricos. Rev. Rene. [Internet] 2006;7(2) [acesso em mai 2012]. Disponível: http://www.revistarene.ufc. br/revista/index.php/revista/article/view/794
14. França CMV. A prevalência de gestantes portadoras de DHEG que apresentaram a síndrome HELLP em uma maternidade de referência de Maceió-AL [dissertação]. Maceió (AL): Universidade Estadual de Ciências da Saúde de Alagoas (UNCISAL); 2010.

15. Coimbra LC, Silva AAM, Mochel EG, Alves MTSSB, Ribeiro VS, Aragão VMF et al. Fatores associados à inadequação do uso da assistência pré-natal. Rev. Saúde Públ. [Internet] 2003;37(4) [acesso em mai 2012]. Disponível: http://dx.doi.org/10.1590/S003489102003000400010

16. Barreto JOM, Nery IS, Mendes YMMB. Mortalidade perinatal: uma análise com enfoque na evitabitabilidade. Cogitare enferm. [Internet] 2011;16(1) [acesso em 20 mai 2013]. Disponível: http://ojs.c3sl.ufpr.br/ojs2/index. php/cogitare/article/download/21117/13943

17. Peracoli JC, Parpinelli MA. Síndromes hipertensivas da gestação: identificação de casos graves. Rev. Bras. Ginecol. Obstet. [Internet] 2005;27(10) [acesso em 20 maio 2013]. Disponível: http://dx.doi.org/10.1590/ S0100-72032005001000010

Cogitare Enferm. 2014 Jan/Mar; 19(1):147-52 